\title{
In Vitro / Ex Vivo Investigation of Modified Utah Electrode Array to Selectively Sense and Pace the Sub-Surface Cardiac \\ His Bundle
}

Ankur R. Shah 1,2, §, Muhammad S. Khan 2, §, Annie M. Hirahara 1,2, Matthias Lange ${ }^{2}$, Ravi Ranjan ${ }^{1,2,3}$, Derek J. Dosdall 1,2,3,4, *

1 Department of Biomedical Engineering,

2 Nora Eccles Harrison Cardiovascular Research and Training Institute,

${ }^{3}$ Division of Cardiovascular Medicine, Department of Internal Medicine

4 Division of Cardiothoracic Surgery, Department of Surgery

The University of Utah, Salt Lake City, UT 84112, USA

$\S$ Authors contributed equally.

*Correspondence:

Dr. Derek J. Dosdall

Associate Professor of Surgery,

Division of Cardiothoracic Surgery, School of Medicine

The University of Utah,

95 S 2000 E, RM 207,

Salt Lake City, UT 84112

Email: derek.dosdall@utah.edu / Ph: (+1) 801-587-2036 
Table of Contents:

Total pages: S1-S9

Figures in the document:

Figure S1. The UEA consists of 100 electrodes on the end of $1.5 \mathrm{~mm}$ long needles arranged in a $10 \times 10$ matrix.

Figure S2. His bundle with standard single electrode lead, SelectSecure 3830 and UEA array.

Figure S3. Raw electrograms recorded through two electrodes of the UEA. (A) Sinus beat with no HB signal.

Figure S4. Nyquist spectrum of selected electrodes that were stimulated at $6 \mathrm{~mA}$ and 8 $\mathrm{mA}$ for $20 \mathrm{~s}$ during in vitro testing.

Figure S5. (A) Real-time continuous HB recording through one of the electrodes of the UEA. Inset shows signals for atrial (A), HIS (H) and ventricle (V). (B) Continuous electrical impedance recording at $1 \mathrm{kHz}$, for 35 seconds at electrode on the $\mathrm{HB}$ interface before pacing. (C) Continuous electrical impedance recording at the same HB location for 35 seconds after pacing the HB.

Figure S6. Continuous electrograms recorded through pseudo-ECG while the UEA implanted in HB-region (A) before and $(B)$ after His pacing. Here ' $P$ ' refers the pacing artifacts. 
Figure S7. Post-stimulation baseline impedance recovery over the period of time in saline. 
(A)

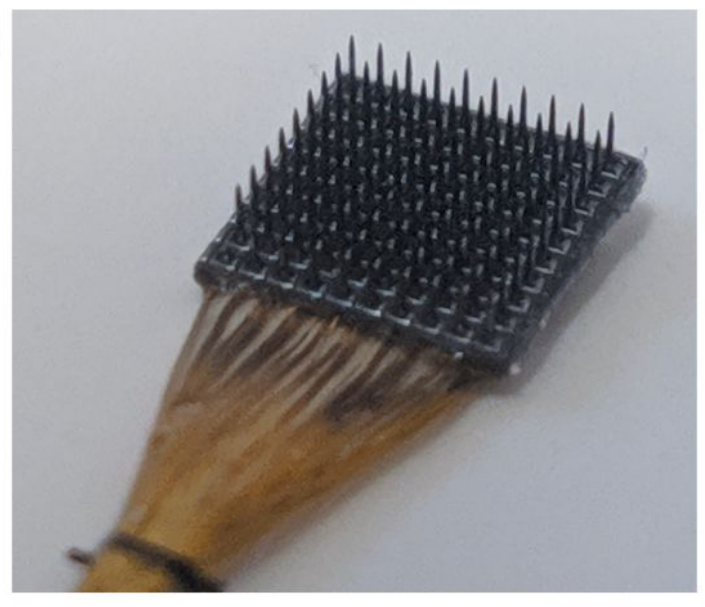

\begin{tabular}{|c|c|c|c|c|c|c|c|c|c|}
\hline & 25 & 39 & 31 & 31 & 35 & 27 & 54 & 17 & \\
\hline 42 & 21 & 27 & 35 & 43 & 30 & 32 & 15 & 30 & 42 \\
\hline 22 & 40 & 47 & 29 & 22 & 12 & 30 & 12 & 33 & 20 \\
\hline 33 & 40 & 22 & 24 & 18 & 26 & 35 & 35 & 37 & 20 \\
\hline 37 & 25 & 23 & 28 & 20 & 25 & 22 & 22 & 31 & 19 \\
\hline 32 & 20 & 40 & 26 & 30 & 16 & 32 & 32 & 10 & 10 \\
\hline 22 & 28 & 28 & 31 & 37 & 24 & 36 & 36 & 42 & 25 \\
\hline 26 & 35 & 53 & 66 & 48 & 35 & 32 & 32 & 20 & 28 \\
\hline 56 & 37 & 48 & 36 & 32 & 72 & 30 & 30 & 32 & 24 \\
\hline & 132 & 24 & 124 & 90 & 65 & 72 & 72 & 20 & \\
\hline
\end{tabular}

Figure S1. The UEA consists of 100 electrodes on the end of $1.5 \mathrm{~mm}$ long needles arranged in a 10x10 matrix. Each electrode needle is insulated along the length and also from the neighboring electrodes. (A) Optical image of the UEA. (B) The mean base impedance magnitude (measure $\mathrm{n}=3$ times) of 96 electrodes recorded in saline at $1 \mathrm{KHz}$ before implantation. For each trial, the impedance spectra were acquired over a frequency range of 0.01 to $100 \mathrm{KHz}$ with a step size of 10 points per decade. Orange shows the broken electrodes and were excluded from further characterization and invivo recordings. 
(A)
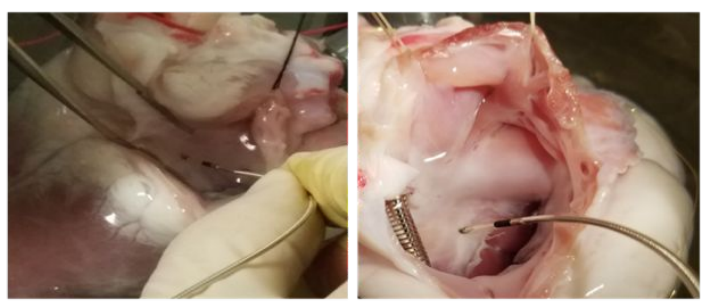

(B)

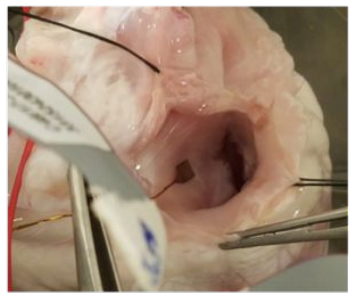

(C)

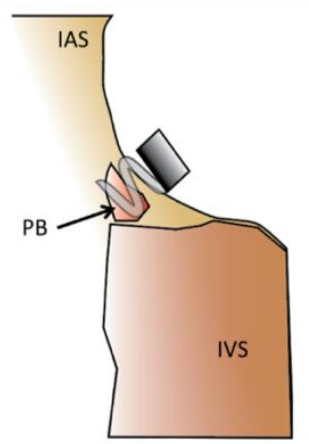

IAS: INTER ATRIALSEPTUM IVS: INTER VENTRICULAR SEPTUM PB: PENETRATING BUNDLE

Structural difference between a 3830 SelectSecure His-Bundle pacing lead (left) and the Utah Electrode Array (right)

Figure S2. His bundle with standard single electrode lead (SelectSecure 3830) and UEA array. (A) Medtronic SelectSecure model 3830 single lead utilized in finding the HIS region (left). Inserted single lead at the His bundle region (right). (B) UEA array inserted in the His region showed high surface area coverage (left). (C) Structural difference between a 3830 SelectSecure HB pacing lead (left) and the UEA (right). Inserted UEA array with option to selectively pace at different sites utilizing multichannel electrodes. 

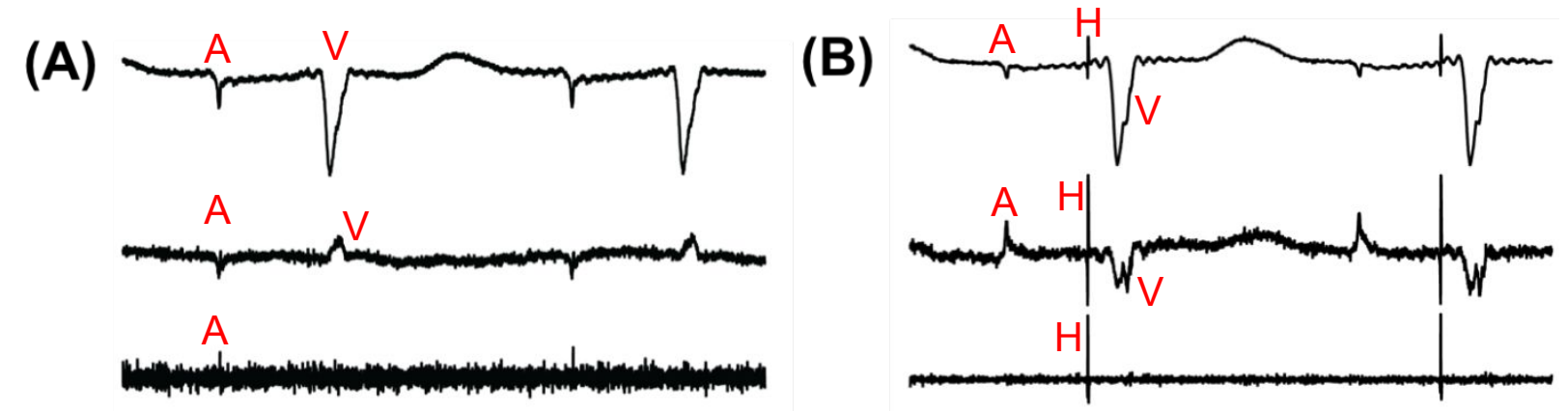

Figure S3. Raw electrograms recorded through two electrodes of the UEA. (A) Sinus beat with no HB signal. (B) Sinus beats with HB signal. HB sensing in sinus (top). Laplacian (middle) magnifies the HIS along with atrial and ventricle. Spatial derivative (bottom) reveals and easily identifiable His signal. A: atrial signal, H: HB signal, V: ventricular signal 


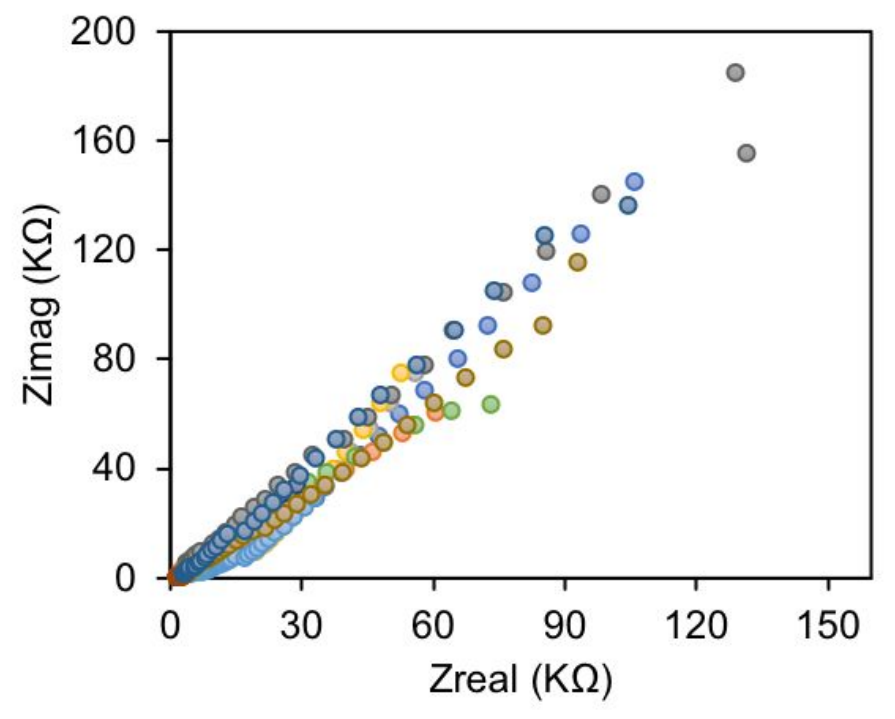

Figure S4. Nyquist spectrum of selected electrodes that were stimulated at $6 \mathrm{~mA}$ and 8 $\mathrm{mA}$ for $20 \mathrm{~s}$ during in vitro testing. 
(A)

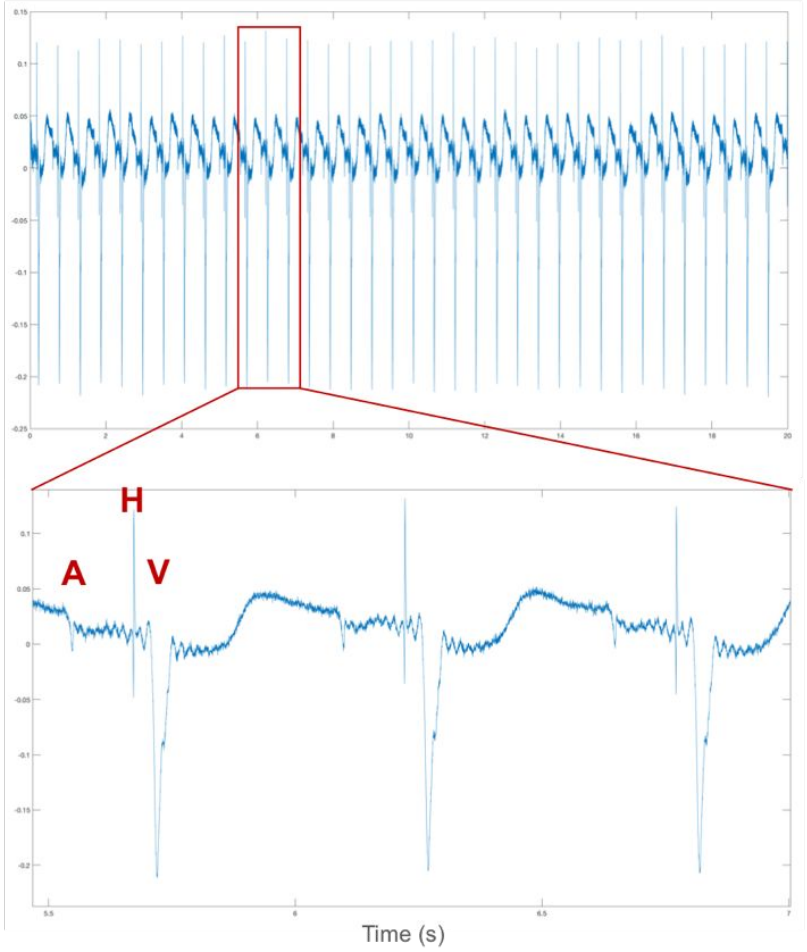

(B)

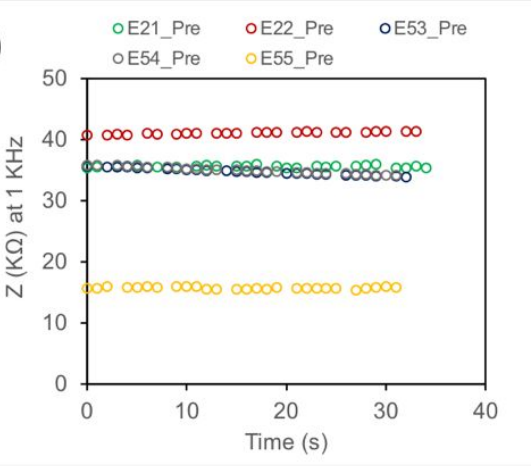

(C)

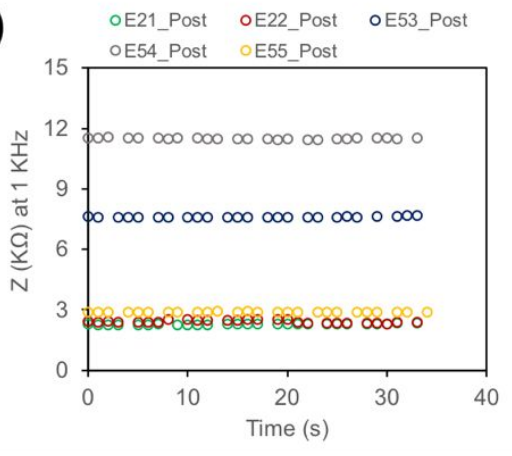

Figure S5. (A) Real-time continuous HB recording through one of the electrodes of the UEA. Inset shows signals for atrial (A), HIS (H) and ventricle (V). (B) Continuous electrical impedance recording at $1 \mathrm{kHz}$, for 35 seconds at electrode on the HB interface before pacing. (C) Continuous electrical impedance recording at the same HB location for $35 \mathrm{sec}$ after pacing the HB. 


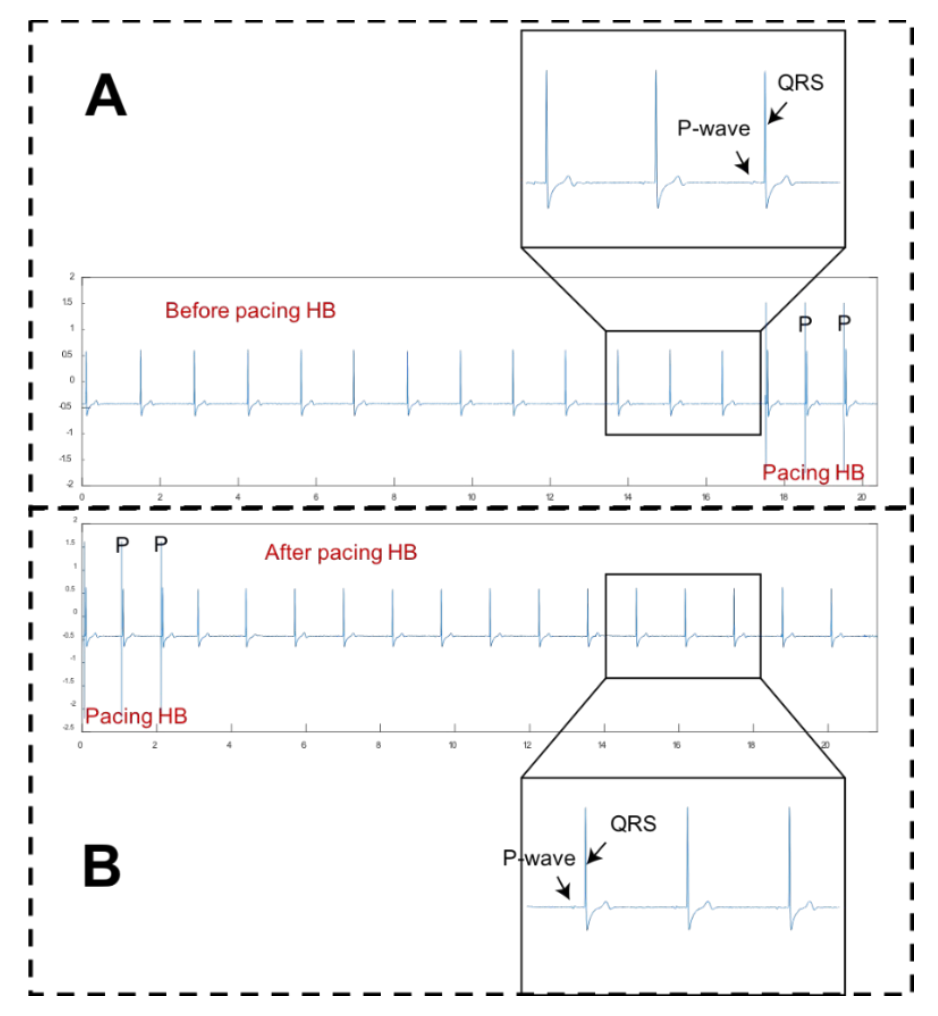

Figure S6. Continuous electrograms recorded through pseudo-ECG while the UEA implanted in HB-region ( $\mathrm{A}$ ) before and $(\mathrm{B})$ after His pacing. Here ' $\mathrm{P}$ ' refers the pacing artifacts. 


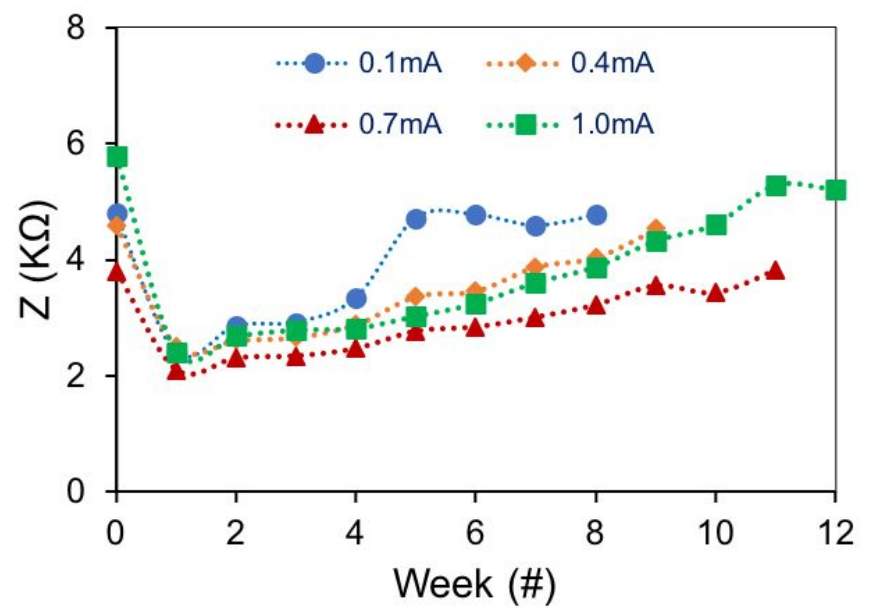

Figure S7. Post-stimulation baseline impedance recovery over the period of time in saline. 